\title{
OGLE-2015-BLG-1459L: The Challenges of Exo-moon Microlensing
}

\author{
K.-H. Hwang ${ }^{1}$ (iD), A. Udalski ${ }^{2}$, I. A. Bond ${ }^{3}$,
}

and

M. D. Albrow ${ }^{4}$ (D) S.-J. Chung ${ }^{1,5}$, A. Gould ${ }^{1,6,7}$, C. Han $^{8}$ (D), Y. K. Jung ${ }^{1}$, Y.-H. Ryu ${ }^{1}$ (D), I.-G. Shin ${ }^{9}$ (D) J. C. Yee ${ }^{9}$ (D) , W. Zhu ${ }^{6}$, S.-M. Cha ${ }^{1,10}$, D.-J. Kim ${ }^{1}$, H.-W. Kim ${ }^{1}$, S.-L. Kim ${ }^{1,5}$, C.-U. Lee ${ }^{1,5}$, D.-J. Lee ${ }^{1}$, Y. Lee ${ }^{1,10}$, B.-G. Park ${ }^{1,5}$, R. W. Pogge ${ }^{6}$ (D) (KMTNet Collaboration),

M. Pawlak ${ }^{2}$, R. Poleski ${ }^{2,6}$ (D), M. K. Szymański ${ }^{2}$, J. Skowron ${ }^{2}$ (D) I. Soszyński ${ }^{2}$, P. Mróz ${ }^{2}$, S. Kozłowski ${ }^{2}$, P. Pietrukowicz ${ }^{2}$ (D),

$$
\text { K. Ulaczyk }{ }^{2}
$$

(OGLE Collaboration),

and

F. Abe ${ }^{11}$, Y. Asakura ${ }^{11}$, R. Barry ${ }^{12}$, D. P. Bennett ${ }^{12}$ (D), A. Bhattacharya ${ }^{12}$, M. Donachie ${ }^{13}$, P. Evans ${ }^{13}$, A. Fukui ${ }^{14}$ (D), Y. Hirao ${ }^{15}$, Y. Itow ${ }^{11}$ (D) K. Kawasaki ${ }^{15}$ (D) N. Koshimoto ${ }^{15}$ (D), M. C. A. Li ${ }^{13}$, C. H. Ling ${ }^{3}$, K. Masuda ${ }^{11}$, Y. Matsubara ${ }^{11}$, S. Miyazaki ${ }^{15}$ (D), Y. Muraki $^{11}$, M. Nagakane ${ }^{15}$, K. Ohnishi ${ }^{16}$, C. Ranc ${ }^{12}$, N. J. Rattenbury ${ }^{13}$ (D), To. Saito ${ }^{17}$, A. Sharan ${ }^{13}$, D. J. Sullivan ${ }^{18}$, T. Sumi ${ }^{15}$, D. Suzuki ${ }^{12,19}$ (D) P. J. Tristram ${ }^{20}$, T. Yamada ${ }^{21}$, T. Yamada ${ }^{15}$, and A. Yonehara ${ }^{21}$

(MOA Collaboration)

${ }^{1}$ Korea Astronomy and Space Science Institute, Daejon 34055, Republic of Korea

${ }^{2}$ Warsaw University Observatory, Al. Ujazdowskie 4, 00-478 Warszawa, Poland

${ }^{3}$ Institute of Natural and Mathematical Sciences, Massey University, Auckland 0745, New Zealand

${ }^{4}$ University of Canterbury, Department of Physics and Astronomy, Private Bag 4800, Christchurch 8020, New Zealand

${ }^{5}$ Korea University of Science and Technology, 217 Gajeong-ro, Yuseong-gu, Daejeon 34113, Republic of Korea

${ }^{6}$ Department of Astronomy, Ohio State University, 140 W. 18th Avenue, Columbus, OH 43210, USA

${ }^{7}$ Max-Planck-Institute for Astronomy, Königstuhl 17, D-69117 Heidelberg, Germany

${ }^{8}$ Department of Physics, Chungbuk National University, Cheongju 28644, Republic of Korea

${ }^{9}$ Harvard-Smithsonian Center for Astrophysics, 60 Garden Street, Cambridge, MA 02138, USA

${ }^{10}$ School of Space Research, Kyung Hee University, Yongin, Kyeonggi 17104, Republic of Korea

${ }^{11}$ Institute for Space-Earth Environmental Research, Nagoya University, Nagoya 464-8601, Japan

${ }^{12}$ Code 667, NASA Goddard Space Flight Center, Greenbelt, MD 20771, USA; david.bennett@nasa.gov

${ }^{13}$ Department of Physics, University of Auckland, Private Bag 92019, Auckland, New Zealand

${ }^{14}$ Okayama Astrophysical Observatory, National Astronomical Observatory of Japan, 3037-5 Honjo, Kamogata, Asakuchi, Okayama 719-0232, Japan

${ }^{15}$ Department of Earth and Space Science, Graduate School of Science, Osaka University, Toyonaka, Osaka 560-0043, Japan

${ }_{17}$ Nagano National College of Technology, Nagano 381-8550, Japan

${ }^{17}$ Tokyo Metropolitan College of Aeronautics, Tokyo 116-8523, Japan

${ }^{18}$ School of Chemical and Physical Sciences, Victoria University, Wellington, New Zealand

${ }^{19}$ Institute of Space and Astronautical Science, Japan Aerospace Exploration Agency, Kanagawa 252-5210, Japan

${ }^{20}$ University of Canterbury Mt. John Observatory, P.O. Box 56, Lake Tekapo 8770, New Zealand

${ }^{21}$ Department of Physics, Faculty of Science, Kyoto Sangyo University, 603-8555 Kyoto, Japan

Received 2017 August 30; revised 2018 April 30; accepted 2018 May 2; published 2018 June 1

\begin{abstract}
We show that dense OGLE and KMTNet I-band survey data require four bodies (sources plus lenses) to explain the microlensing light curve of OGLE-2015-BLG-1459. However, these can equally well consist of three lenses and one source (3L1S), two lenses and two sources (2L2S), or one lens and three sources (1L3S). In the 3L1S and 2L2S interpretations, the host is a brown dwarf and the dominant companion is a Neptune-class planet, with the third body (in the 3L1S case) being a Mars-class object that could have been a moon of the planet. In the 1L3S solution, the light curve anomalies are explained by a tight (five stellar radii) low-luminosity binary source that is offset from the principal source of the event by $\sim 0.17$ au. These degeneracies are resolved in favor of the $1 \mathrm{~L} 3 \mathrm{~S}$ solution by color effects derived from comparison to MOA data, which are taken in a slightly different $(R / I)$ passband. To enable current and future (WFIRST) surveys to routinely characterize exo-moons and distinguish among such exotic systems requires an observing strategy that includes both a cadence faster than 9 minute $^{-1}$ and observations in a second band on a similar timescale.
\end{abstract}

Key words: gravitational lensing: micro - planetary systems

\section{Introduction}

The eight planets of the solar system harbor an amazing diversity of moons. Two planets have no moons, while Jupiter has four major moons that Johannes Kepler already realized constitute a mini-solar system obeying his Third Law. Some moons are mostly ice while others are entirely composed of rock. A few have atmospheres, lakes, geysers, and other features. Some were captured and others formed in situ. It is even speculated that some moons harbor life.

While the number of confirmed or highly probable exo-planets is several hundred times greater than the eight solar system planets, the situation for exo-moons is the reverse: no clear discoveries (but see Teachey et al. 2018). Exo-moons generate almost zero signal in Doppler studies of host stars because the 
barycenter of the planet-moon system follows almost exactly the same orbit as an isolated planet. The transit method is more sensitive to exo-moons: Earth's moon would give rise to a transit signal that is $\sim 7 \%$ of Earth's. However, because the Earth signal is itself near the detection limit for a single transit for the Kepler satellite, similar moons would be most easily detected for very close-in planets that had many transits during the mission (see Kipping et al. 2015 and references therein).

The microlensing method may have greater potential to detect exo-moons. Microlensing occurs when a massive body (star, planet, etc.) becomes closely aligned with a more distant source star. The gravity of the foreground object (lens) bends the source light and thereby magnifies it. The changing magnification gives rise to a "microlensing event." In the case of planet-star (or more complicated) systems, the light curve can be complex, thus revealing the presence, geometry, and masses of multiple components.

The detectability of an isolated object (be it lunar, planetary, or stellar mass) is governed by microlensing's characteristic angular scale, the Einstein radius

$$
\theta_{\mathrm{E}}=\sqrt{\kappa M \pi_{\mathrm{rel}}} ; \quad \kappa \equiv \frac{4 G}{c^{2} \mathrm{au}} \simeq 8.1 \frac{\mathrm{mas}}{M_{\odot}},
$$

where $M$ is the lens mass and $\pi_{\text {rel }} \equiv \mathrm{au}\left(D_{L}^{-1}-D_{S}^{-1}\right)$ is the lenssource relative parallax. Hence, the cross section for microlensing detection scales $\theta_{\mathrm{E}} \sim M^{1 / 2}$.

If the lens consists of two bodies, then in the limit that the separation between them is $s \gg 1$, the light curve can appear as two isolated "bumps." In this case, the requirement for detecting the second bump (and second body) is that the source passes within roughly $\theta_{\mathrm{E}, p}=\sqrt{q} \theta_{\mathrm{E}}$ of the planet, where $q$ is the planetstar mass ratio, e.g., OGLE-2016-BLG-0263 (Han et al. 2017). This mildly favors high-mass planets, but because there are more low-mass than high-mass planets, microlensing planet detections are almost uniform in $\log q$ (Mróz et al. 2017). As the planet gets closer to the Einstein radius of the star, it gives rise to a growing caustic structure (contours of formally infinite magnification for point sources) that becomes much larger than its own Einstein radius. Hence, over half of detections have projected separations very close to the star's Einstein radius, $0.8<s<1.25$ (Mróz et al. 2017). The scaling of the caustics with $q$ is either $q^{1 / 3}, q^{1 / 2}$, or $q$ depending on the type of caustic (Gaudi 2012).

An exo-moon differs from a planet only in that it lies in the extreme low-mass regime. For a lens lying halfway toward the Galactic bulge (where all microlensing planet searches are conducted), $\theta_{\mathrm{E}}=1.7 \mu \mathrm{as}\left(M / M_{\oplus}\right)^{1 / 2}$. For exo-moons, this is likely to be smaller than the angular radius of the source, typically $\theta_{*} \sim 0.5 \mu$ as (e.g., $\theta_{\mathrm{E}}=0.17 \mu$ as for $M=0.01 M_{\oplus}, D_{L}=4$ $\mathrm{kpc}, D_{S}=6 \mathrm{kpc}$ ). Thus, an isolated exo-moon would magnify only a small fraction of the source, making it difficult or impossible to detect. However, just as the planet generates a larger effect if it is close to the star's Einstein radius, its moon can likewise have an outsized effect on the planet's caustic. Thus, one expects that exo-moons would be most easily detectable by distorting the caustic due to its host planet (although in principle they can also change the topology of the caustic, e.g., by adding extra cusps). The topology variations of three-body lens systems are discussed in detail by Daněk \& Heyrovský (2015a, 2015b) and Song et al. (2014). Han \& Han (2002), Han (2008), Liebig \& Wambsganss (2010), and Chung \& Ryu (2016) have studied exomoon-specific features of microlensing light curves and find a wide variety of light curve morphologies.
Here, we investigate the microlens OGLE-2015-BLG-1459L and show that based on the $I$-band light curve alone, it is an exo-moon candidate. However, we also show that there are two alternate solutions. In the first, there is only a host and planet but no exo-moon. The additional anomaly that could be attributed to an exo-moon is then attributed to a second source (companion to the primary source). In the second, both the main and secondary anomalies are attributed to the companion sources, so that there is a triple-source system but only a single lens. We then resolve these degeneracies in favor of the triplesource solution by measuring the evolving color of the event. Finally, we discuss the wider implications of this event for the practical study of exo-moons with microlensing.

\section{Observations}

On 2015 June $25\left(\mathrm{HJD}^{\prime}=\mathrm{HJD}-2450000=7199.1\right)$, the Optical Gravitational Lensing Experiment (OGLE; Udalski et al. 2015) collaboration alerted the microlensing community to OGLE-2015BLG-1459 via its Early Warning system (Udalski et al. 1994) ${ }^{22}$ based on observations from their $1.3 \mathrm{~m}$ telescope in Chile, which were carried out with cadence $\Gamma=3 \mathrm{hr}^{-1}$, primarily in $I$ band. The event lies at (R.A., decl.) $=(18: 00: 50.40,-28: 40: 15.7)$, corresponding to $(l, b)=(1.92,-2.73)$. From Chile, the event appears to be a simple point lens (1L1S) event that peaks relatively faint $I_{\text {peak }} \sim 18$. However, the Korea Microlensing Telescope Network (KMTNet; Kim et al. 2016) independently observed this field from three different sites during their commissioning-year observations. KMTNet also observed primarily in $I$ band, with cadence $\Gamma=7 \mathrm{hr}^{-1}$ from each of its three $1.6 \mathrm{~m}$ telescopes in Chile (KMTC), South Africa (KMTS), ${ }^{23}$ and Australia (KMTA). KMTA data showed a strong anomaly just after the peak (see Figure 1). The OGLE and KMTNet data were reduced using their difference image analysis (DIA; Alard \& Lupton 1998) photometry pipelines (Woźniak 2000 and Albrow et al. 2009, respectively).

The Microlensing Observations in Astrophysics (MOA) collaboration also took data of this field using their $1.8 \mathrm{~m}$ telescope at Mt. John, New Zealand, which employs a broad $R / I$ filter. These data were originally believed to be too low quality to be of use and were not initially reduced. And indeed, even after careful rereduction using a variant of DIA (Bond et al. 2017), they do not significantly constrain any of the geometric parameters. However, because they are in a slightly different passband, they do constrain the flux parameters, which proves crucial to resolving the degeneracy between the models (Section 5).

It is well known that the photometric errors that come from the photometry reduction algorithms are imperfect and can underestimate the true errors. Therefore, we rescale the error bars for each data set using a variant of the method in Yee et al. (2012), which implicitly assumes that the underlying errors follow a Gaussian distribution. For this event, the vast majority of the data are taken when the event is below sky, but the data on the night of the anomaly are above sky. We cannot assume that the photometric algorithms adequately account for this transition. Therefore, we renormalize the error bars on the peak night $\left(\mathrm{HJD}^{\prime}=7200\right)$ separately from the data for the rest of the event such that the total $\chi^{2}$ per degree of freedom is 1 . The error renomalization factors are given in Table 1.

\footnotetext{
22 http://ogle.astrouw.edu.pl/ogle4/ews/ews.html

${ }^{23}$ KMTS was down for engineering during all but the tail end of the event and so the data are not used here.
} 

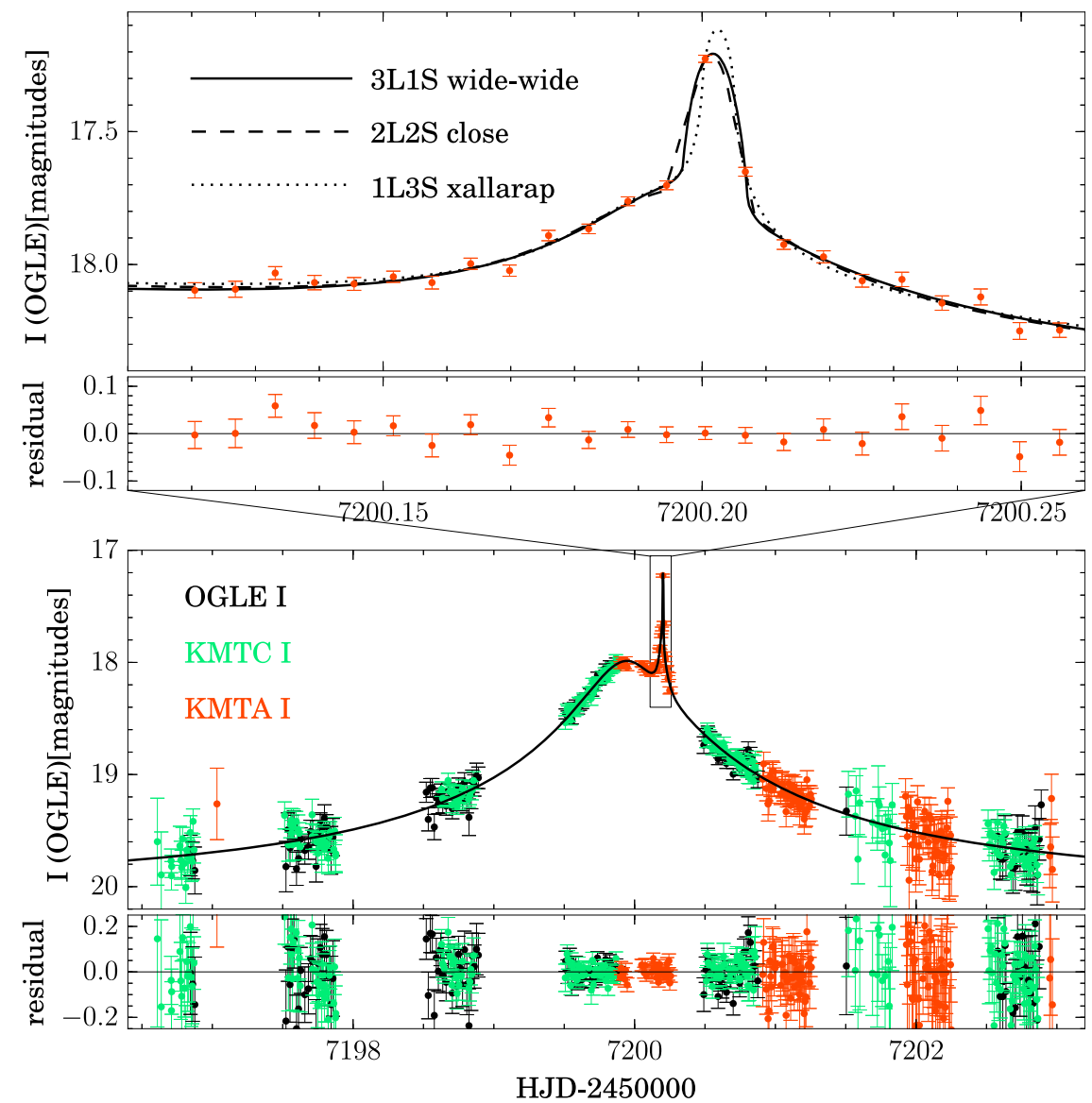

Figure 1. OGLE-2015-BLG-1459 light curve together with three models that fit the $I$-band data from OGLE and KMTNet equally well. One model (3L1S) contains three bodies: a brown dwarf host, a Neptune-class planet, and a Mars-class object that may orbit the "Neptune." The second model (2L2S) contains a brown dwarf host and a Neptune-class planet. The third model (1L3S) has a single lens that microlenses a triple-source system. Actually, there are four variants of the planet/moon model and two variants of the planet model, but the remaining solutions look almost identical to those shown (wide-wide and close, respectively).

\section{Analysis}

The anomaly (Figure 1) consists of two features: a broad bump and an outlying point at $\mathrm{HJD}^{\prime}=7200.200$, which is $\sim 0.45$ mag (a factor of $\sim 1.5$ in flux) brighter than the two neighboring points taken $\sim 9$ minutes earlier and later. Figure 2 shows the observations and subtracted images at these epochs. As we show below, each of these features is subject to two interpretations, i.e., each can indicate the presence of an additional source or an additional lens component.

\subsection{Models with Three Bodies}

To facilitate the analysis, we begin by fitting just the OGLE and KMTNet data and by temporarily removing the outlying point. That is, we fit for models with a two-body lens and a single source star (2L1S) and models with a single lens and two source stars (1L2S).

We first fit the pruned light curve with a 2L1S model. Such models have three parameters describing the underlying stellar event (the impact parameter, $u_{0} \theta_{\mathrm{E}}$, the time of closest approach, $t_{0}$, the Einstein crossing time, $t_{\mathrm{E}}$ ), three parameters describing the planet (its mass ratio, $q$, its projected separation, $s \theta_{\mathrm{E}}$, and the angle between the source trajectory and the planet-star axis, $\alpha$ ), and the normalized source radius $\rho=\theta_{*} / \theta_{\mathrm{E}}$. A thorough search of parameter space yields two solutions ("close" and "wide," $s<1$ and $s>1$, respectively), which are related by the well known
Table 1

Parameters for Scaling Data Error bars

\begin{tabular}{lcc}
\hline \hline Telescope & Number & $k$ \\
\hline OGLE & 2088 & 1.30 \\
MOA & 2186 & 1.09 \\
MOA (peak) & 19 & 0.98 \\
KMTC & 1399 & 1.63 \\
KMTA & 1091 & 2.61 \\
KMTA (peak) & 34 & 1.50 \\
\hline
\end{tabular}

$s \leftrightarrow s^{-1}$ degeneracy for central caustics (Griest \& Safizadeh 1998; Dominik 1999).

We then fit the same light curve to a single lens that microlenses a binary source (1L2S). The minimum requirement for such a fit, which we employ here, is five geometric parameters: $t_{\mathrm{E}}$, plus two pairs of $\left(t_{0}, u_{0}\right)_{i}$, one for each source, $i=1,2$. In addition, this model requires an extra flux parameter $q_{F}$, which is the ratio of source fluxes in $I$ band (which is then treated as being the same at all observatories).

Figure 3 shows the light curve in the region of the cusp approach together with these two models, i.e., the $1 \mathrm{~L} 2 \mathrm{~S}$ model and the close 2L1S models (the wide model being almost identical). Both models explain the broad bump in the anomaly. However, neither can explain the outlying point. 

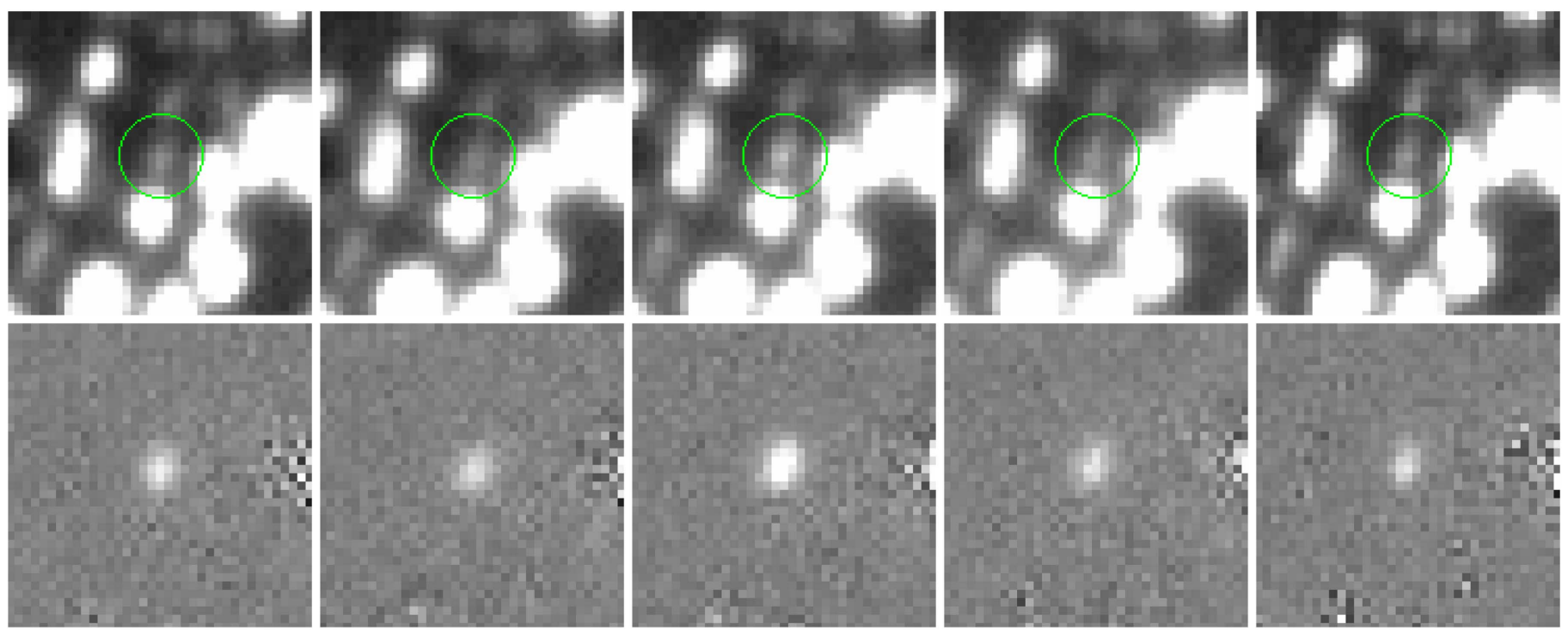

Figure 2. Actual (upper panels) and subtracted (lower panels) images from KMTA for the five observations centered on the "outlying point" (i.e., for HJD' $\equiv$ HJD $-2457200.0=0.188,0.194,0.200,0.207$, and 0.213 , left to right).
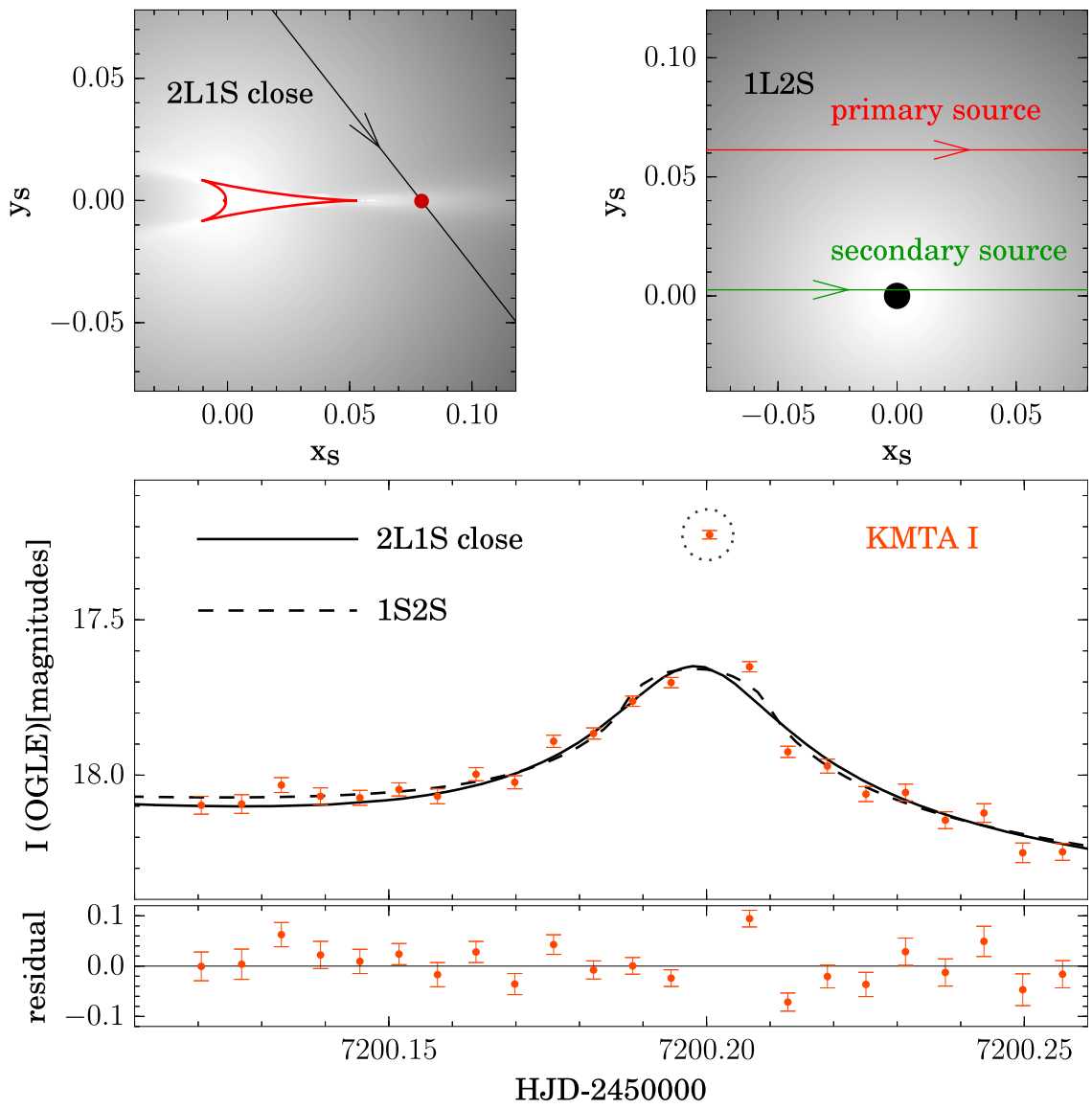

Figure 3. Best-fit binary-lens (2L1S) and binary source (1L2S) models to all the data shown in Figure 1 except that the high point at 7200.200 (dotted circle) is excluded. Both models are reasonably good, with the main deviation in KMTA data explained either by a typical four-pronged "central caustic" due to a planet (2L1S) or a second source that is 1.7 mag fainter than the primary but lies much closer to the path of the source. There are two degenerate $2 \mathrm{~L} 1 \mathrm{~S}$ models, which yield very similar light curves. Hence, only the "close" solution is shown.

\subsection{Models with Four Bodies}

Although in principle, a single outlier could be due to a number of different phenomena, Occam's razor tells us it is far more likely that this is due to an additional microlensing effect. There are three ways to modify these models to account for the outlying point, all of which require adding an additional body to the model.

In the first of these, one adds an additional source to the $1 \mathrm{~L} 2 \mathrm{~S}$ model to obtain $1 \mathrm{~L} 3 \mathrm{~S}$. The additional source is positioned so that it is transited by the lens at the time of the 
spike. The extremely short duration of the spike (combined with its modest peak amplitude) is then attributed to the extreme faintness of this third source. In fact, both additional sources must be quite faint, a point to which we return below. This minimalist version of the $1 \mathrm{~L} 3 \mathrm{~S}$ model requires three more geometric parameters $\left(t_{0}, u_{0}, \rho\right)_{3}$, as well as an additional flux ratio parameter $q_{F, 2}$.

In the second model, one adds a second source to the $2 \mathrm{~L} 1 \mathrm{~S}$ model to produce $2 \mathrm{~L} 2 \mathrm{~S}$. The spike is then explained by the second source passing over the cusp at almost exactly the same time (within 2 minutes) that the first source passes over the "magnification spike" that extends away from the tip of the caustic shown in Figure 3. This model requires the same three additional parameters as the one described in the previous paragraph.

In the third model, one adds a third lens to the $2 \mathrm{~L} 1 \mathrm{~S}$ model to produce $3 \mathrm{~L} 1 \mathrm{~S}$. In this case, the spike is explained by the third body distorting (lengthening) the cusp seen in Figure 3 so that the source passes directly over it. This requires three additional geometric parameters relative to the $2 \mathrm{~L} 1 \mathrm{~S}$ model, i.e., an additional pair of $(s, q)_{2}$ and an angle $\psi$ between the binary axis and the line connecting the third body to the primary. In this case, there are four degenerate solutions, i.e., a close-wide degeneracy (Griest \& Safizadeh 1998; Han et al. 2013) for each of the two low-mass companions (see Figure 4).

Note that this does not represent an exhaustive search for triple lens solutions (see Han et al. 2017, for an example of such a search). However, Figure 8 shows that these models explain all of the major features of the light curve. While there may be other 3L1S models that also fit the data, there is no indication (e.g., via significant residuals) that an alternative triple lens model would give an improved fit. We will revisit to this point in Section 5.

As shown in Figure 1 and quantified in Table 2, each of the three of these basic models have variants that fit the data approximately equally well. That is, the $\chi^{2}$ of the "xallarap" variant of the $1 \mathrm{~L} 3 \mathrm{~S}$ model (see Section 4.1) is comparable to the $\chi^{2}$ of the better of the two 2L2S models and the best of the four 3L1S models. Note that we do not show the parameter values for the various models listed in Table 2 because these are very similar to the ones that we discuss in Section 5.

We first investigate the physical properties of these three models in Section 4. Then, in Section 5, we ask how we can distinguish between the three models given that the formal statistical difference between the models compared to OGLE and KMTNet data is insignificant.

\section{Physical Properties}

\section{1. $1 L 3 S$}

The sources of the 1L3S solution likely form a gravitationally bound hierarchical triple. In the static version of this solution, the primary is responsible for the "main event," and the two fainter sources are each responsible for one of the anomaly features. As mentioned in Section 3, the two fainter sources induce peaks at almost exactly the same time, implying that they are projected close to each other within the Einstein ring $\Delta u=0.0031$, where $\Delta \boldsymbol{u} \equiv\left[\left(t_{0,2}-t_{0,3}\right) / t_{\mathrm{E}}, u_{0,2}-u_{0,3}\right]$ (see Table 3 , below). This is actually only a few times larger than the normalized source size $\rho=0.0006$, meaning that these two stars form an extremely tight binary (unless they are seen in an extremely unlikely chance projection). This in turn implies that treating this binary as static is not a reasonable approximation, because the two components are likely moving at several hundred kilometers per second.
We therefore fit them to a standard xallarap (binary-source motion) model, which allows for circular motion characterized by four parameters, i.e., the three-dimensional separation $\chi_{\mathrm{E}}$ (scaled to $\theta_{\mathrm{E}}$ ), and an arbitrary inclination, orientation, and phase of the orbit (see Figure 5 as well as Table 4, below). To determine the period, we assume a total binary mass of $0.5 M_{\odot}$ and a tertiary source radius $R_{3}=0.2 R_{\odot}$ (see below), adopt $\rho_{3}=0.0006$, and then apply Kepler's Third Law. This model hardly improves $\chi^{2}$, but the parameters are quite reasonable. It is also the case that most (perhaps all) very close binaries are in hierarchical triples, which may point to a Lidov-Kozai origin (Fabrycky \& Tremaine 2007). Thus, this solution is in every respect quite reasonable.

Its only "bizarre" feature is that both sources in the compact binary are quite faint, lying $\Delta I_{2}=9$ and $\Delta I_{3}=10$ below the clump of the color-magnitude diagram, where $\Delta I_{i} \equiv I_{s, i}-I_{\text {clump }}$ and $I_{\text {clump }}$ is the magnitude of the clump. These would be the two faintest sources ever reliably measured in a microlensing event. However, given that we see two short-lived perturbations peaking at nearly the same time, it is not particularly surprising that they would be due to a close, faint binary. If the sources were brighter, their flux would have been detectable over a broader portion of the light curve.

\section{2. $2 L 2 S$}

In terms of the physical characteristics of the lens system, the $2 \mathrm{~L} 2 \mathrm{~S}$ and $3 \mathrm{~L} 1 \mathrm{~S}$ are very similar, except that the latter contains an additional, very low-mass object (see Tables 5 and 6, below). In both cases, the mass ratio of the (principal) planet to the host is $q \sim 3 \times 10^{-3}$ and the lens-source relative proper

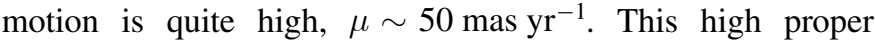
motion implies a very nearby lens, which (given the inverse relation between $M$ and $\pi_{\text {rel }}$ at fixed $\theta_{\mathrm{E}}$ specified by Equation (1)) implies a very low host mass. In both cases, this leads to a brown-dwarf (BD) host orbited by a sub-Saturn (probably Neptune-class) planet. We follow through this reasoning in some detail for $2 \mathrm{~L} 2 \mathrm{~S}$ and then briefly recount the minor differences for $3 \mathrm{~L} 1 \mathrm{~S}$.

In all solutions (1L3S, 2L2S, 3L1S), the (total) source flux is about $f_{s, \text { ogle }} \simeq 0.055$ (normalized to $I=18$ ) and the mean color of the source(s) (determined from regression) is $\Delta(V-I)=$ -0.14 mag blueward of the clump. For the multi-source solutions, we lack separate measurements for the colors of the different sources because the secondary (and possibly tertiary) do not contribute substantially to the total flux during times when there are $V$ data. Nevertheless, because the total flux lies $\sim 4.5 \mathrm{mag}$ below the clump, and is relatively red, we can assume that both (or all three) of the sources lie on a single main-sequence isochrone. We approximate the color-magnitude relation on the main sequence as $\Delta M_{I}=2.4 \Delta(V-I)_{0}$. Then, from the measured flux ratio of the 2L2S model $q_{F}=0.189$, we can infer that the secondary source is $\Delta[I,(V-I)]=(6.5,0.51)$ fainter and redder than the clump.

It is the secondary source that is important in $2 \mathrm{~L} 2 \mathrm{~S}$ because this is the source that transits the cusp, and for which there is a normalized source radius measurement, $\rho_{2}=0.73 \pm 0.13$. We combine the above estimate of the source position relative to the clump with the dereddened clump centroid $[(V-I), I]_{0, \text { clump }}=$ (1.06, 14.38) (Bensby et al. 2013; Nataf et al. 2013), the VIK color-color relation of Bessell \& Brett (1988) and the color/ surface-brightness relation of Kervella et al. (2004), to obtain 

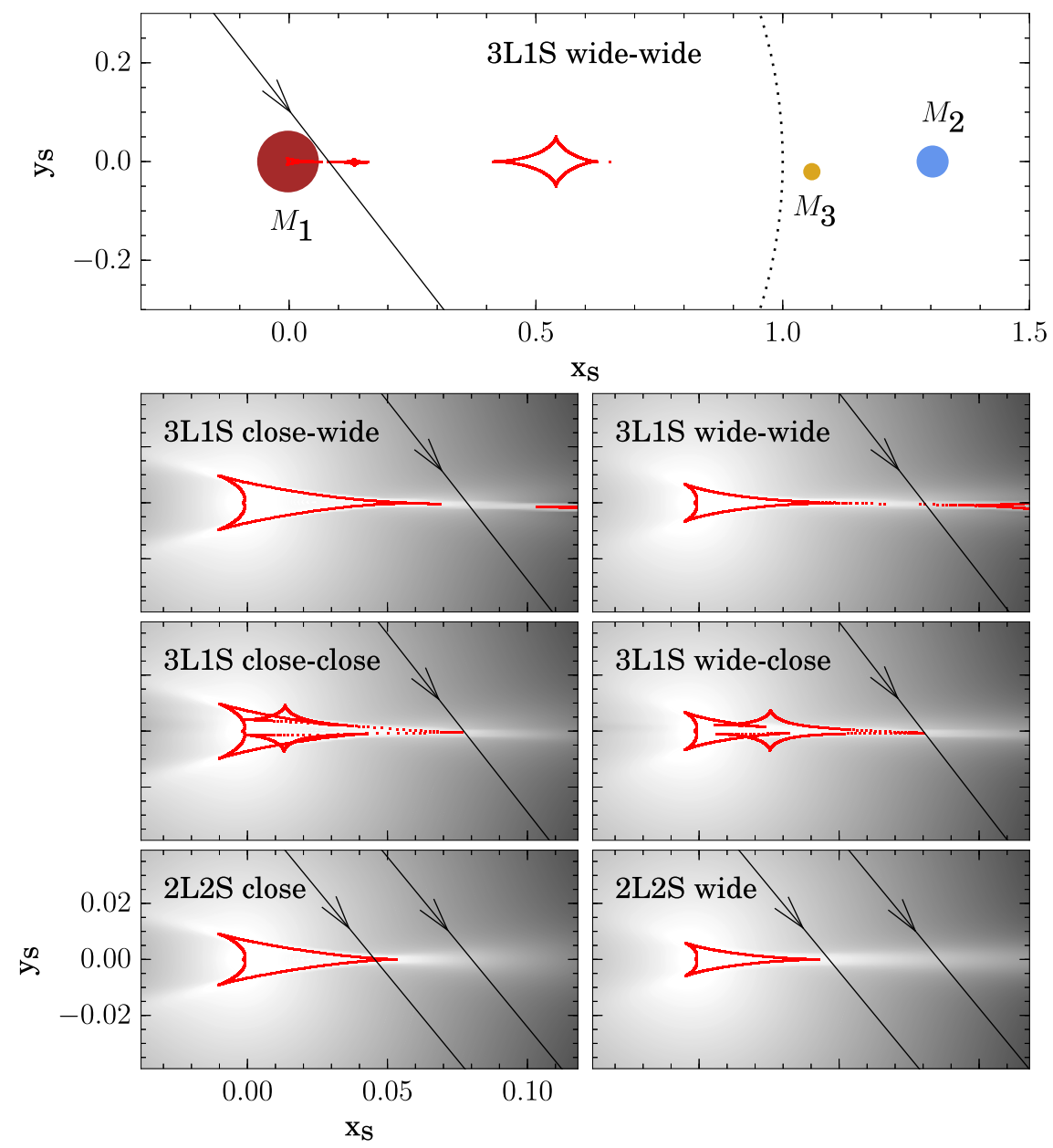

Figure 4. Caustic geometries of the four $3 \mathrm{~L} 1 \mathrm{~S}$ solutions are shown in the middle four panels. In each case, the high point at HJD 7200.200 is explained by the presence of a third body that, because it is roughly aligned with the planet-star axis, "extends" the caustic caused by the planet in Figure. 3. Upper panel is a zoom-out of the wide-wide solution, showing the full geometry. In the bottom two panels, the two 2L2S solutions are shown. In these cases, the high point at HJD 7200.200 is explained by a second source passing over the cusp seen in the upper-left panel of Figure 3.

Table 2

Comparison of $\chi^{2}$ for $I$-band Models

\begin{tabular}{llccc}
\hline \hline Model & Variant & $\chi^{2} /$ dof & $N_{\text {params }}{ }^{\text {a }}$ & $N w / \Delta I>0.3^{\mathrm{b}}$ \\
\hline 1L3S & xallarap & $4605.9 / 4598$ & 13 & 438 \\
1L3S & static & $4608.2 / 4599$ & 12 & 439 \\
\hline 2L2S & close & $4603.8 / 4600$ & 11 & 440 \\
2L2S & wide & $4618.7 / 4600$ & 11 & 440 \\
\hline 3L1S & wide-wide & $4604.7 / 4601$ & 10 & 437 \\
3L1S & wide-close & $4604.8 / 4601$ & 10 & 434 \\
3L1S & close-close & $4608.2 / 4601$ & 10 & 438 \\
3L1S & close-wide & $4608.3 / 4601$ & 10 & 438 \\
\hline
\end{tabular}

Notes.

${ }^{\mathrm{a}}$ The number of parameters of the model.

$\mathrm{b}$ The number of data points $>0.3 \mathrm{mag}$ above baseline.

$\theta_{*}=0.48 \mu$ as (Yoo et al. 2004). This implies

$\theta_{\mathrm{E}}=\frac{\theta_{*}}{\rho}=0.67 \pm 0.12$ mas $\quad \mu=\frac{\theta_{\mathrm{E}}}{t_{\mathrm{E}}}=48 \pm 9 \mathrm{mas} \mathrm{yr}^{-1}$.
Table 3

Parameters for Static 1L3S Model

\begin{tabular}{lr}
\hline \hline & \multicolumn{1}{c}{1 L3S static } \\
\hline$\chi^{2} /$ dof & $6803.83 / 6803$ \\
$t_{0}\left(\mathrm{HJD}^{\prime}\right)$ & $7199.946 \pm 0.004$ \\
$u_{0}$ & $0.065 \pm 0.005$ \\
$t_{\mathrm{E}}$ & $4.921 \pm 0.291$ \\
$\rho_{*}$ & $\ldots$ \\
$t_{0,2}\left(\mathrm{HJD}^{\prime}\right)$ & $7200.193 \pm 0.002$ \\
$u_{0,2}\left(10^{-3}\right)$ & $2.638 \pm 0.676$ \\
$\rho_{*, 2}\left(10^{-3}\right)$ & $4.503 \pm 1.525$ \\
$q_{F, 2}$ & $0.014 \pm 0.003$ \\
$t_{0,3}\left(\mathrm{HJD}^{\prime}\right)$ & $7200.202 \pm 0.001$ \\
$u_{0,3}\left(10^{-3}\right)$ & $0.281 \pm 0.165$ \\
$\rho_{*, 3}\left(10^{-3}\right)$ & $0.631 \pm 0.229$ \\
$q_{F, 3}$ & $0.006 \pm 0.001$ \\
$q_{F, R}(\mathrm{MOA})$ & $0.006 \pm 0.001$ \\
$f_{s}(\mathrm{OGLE})$ & $0.056 \pm 0.004$ \\
$f_{b}(\mathrm{OGLE})$ & $0.100 \pm 0.004$ \\
\hline
\end{tabular}

The proper motion implies that the lens must be nearby. For example, the lens cannot be in the Galactic bulge at $D_{L} \sim 8 \mathrm{kpc}$ because then the lens-source relative velocity 

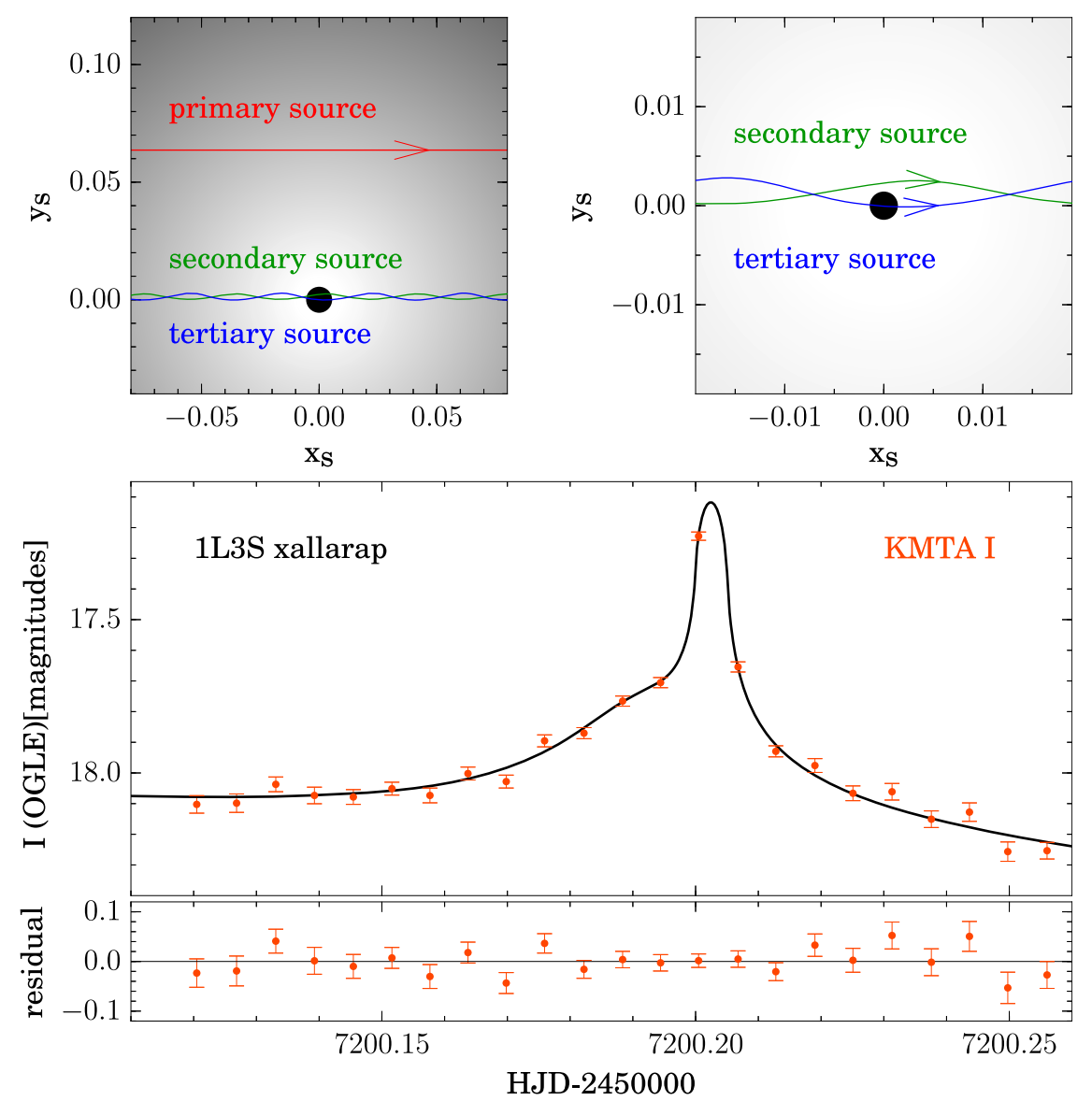

Figure 5. Light curve and geometry for "xallarap" version of 1L3S, in which the secondary and tertiary sources are modeled as being in a circular orbit $4.5 \mathrm{hr}$ orbit.

Table 4

Parameters for 1L3S Xallarap Model

\begin{tabular}{lc}
\hline \hline & 1L3S xallarap \\
\hline$\chi^{2} /$ dof & $6800.96 / 6802$ \\
$t_{0}\left(\mathrm{HJD}^{\prime}\right)$ & $7199.945 \pm 0.004$ \\
$u_{0}$ & $0.067 \pm 0.005$ \\
$t_{\mathrm{E}}$ & $4.913 \pm 0.320$ \\
$\rho_{*}$ & $\cdots$ \\
$t_{0,(2,3)}\left(\mathrm{HJD}^{\prime}\right)$ & $7200.198 \pm 0.001$ \\
$u_{0,(2,3)}\left(10^{-3}\right)$ & $1.406 \pm 0.298$ \\
$\rho_{*, 2}\left(10^{-3}\right)$ & $0.584_{-0.203}^{+1.150}$ \\
$\rho_{*, 3}\left(10^{-3}\right)$ & 0.6 (fixed) \\
$q_{F, 2}$ & $0.0145 \pm 0.0022$ \\
$q_{F, 3}$ & $0.0067 \pm 0.0011$ \\
$q_{F, R}(\mathrm{MOA})$ & $0.0056 \pm 0.0009$ \\
$\chi_{E, X}$ & $0.0021 \pm 0.0008$ \\
$\chi_{E, Y}$ & $0.0020 \pm 0.0006$ \\
$\alpha$ & $270.79 \pm 14.13$ \\
$\delta$ & $-32.11 \pm 29.98$ \\
$f_{s}(\mathrm{OGLE})$ & $0.056 \pm 0.005$ \\
$f_{b}(\mathrm{OGLE})$ & $0.100 \pm 0.005$ \\
\hline
\end{tabular}

would be $\mu_{\text {rel }} D_{L} \sim 1800 \mathrm{~km} \mathrm{~s}^{-1}$, which would imply that either the lens or source was not bound to the Galaxy. Therefore, it must be relatively close to the Sun. Even if the lens is from the quite sparse Galactic halo population, with typical transverse speed of $v_{\perp} \sim 200 \mathrm{~km} \mathrm{~s}^{-1}$, it lies at $D_{L}=v_{\perp} / \mu_{\text {rel }} \sim 0.9 \mathrm{kpc}$. Stars in the thick disk or thin disk
Table 5

Parameters for 2L2S Models

\begin{tabular}{lrr}
\hline \hline & \multicolumn{2}{c}{ 2L2S } \\
\cline { 2 - 3 } & \multicolumn{1}{c}{ close } & \multicolumn{1}{c}{ wide } \\
\hline$\chi^{2} /$ dof & $6808.45 / 6804$ & $6825.04 / 6804$ \\
$t_{0}\left(\mathrm{HJD}^{\prime}\right)$ & $7199.941 \pm 0.005$ & $7199.914 \pm 0.009$ \\
$u_{0}$ & $0.069 \pm 0.003$ & $0.066 \pm 0.004$ \\
$t_{\mathrm{E}}$ & $4.613 \pm 0.201$ & $5.106 \pm 0.231$ \\
$s$ & $0.857 \pm 0.009$ & $1.260 \pm 0.014$ \\
$q\left(10^{-3}\right)$ & $2.374 \pm 0.207$ & $2.068 \pm 0.209$ \\
$\alpha$ & $0.890 \pm 0.008$ & $0.875 \pm 0.009$ \\
$\rho_{*}\left(10^{-3}\right)$ & $0.688 \pm 0.595$ & $1.795 \pm 0.871$ \\
$t_{0,2}\left(\mathrm{HJD}^{\prime}\right)$ & $7200.057 \pm 0.010$ & $7200.049 \pm 0.008$ \\
$u_{0,2}$ & $0.039 \pm 0.003$ & $0.036 \pm 0.003$ \\
$\rho_{*, 2}\left(10^{-3}\right)$ & $0.542 \pm 0.161$ & $0.457 \pm 0.164$ \\
$q_{F}$ & $0.181 \pm 0.077$ & $0.396 \pm 0.090$ \\
$q_{F, R}(\mathrm{MOA})$ & $0.177 \pm 0.072$ & $0.392 \pm 0.090$ \\
$f_{s}(\mathrm{OGLE})$ & $0.061 \pm 0.003$ & $0.054 \pm 0.003$ \\
$f_{b}(\mathrm{OGLE})$ & $0.095 \pm 0.003$ & $0.101 \pm 0.003$ \\
\hline
\end{tabular}

populations are 50 or 500 times more common than halo stars, but typically have $v_{\perp} \sim 100 \mathrm{~km} \mathrm{~s}^{-1}$ or $v_{\perp} \sim 50 \mathrm{~km} \mathrm{~s}^{-1}$, meaning the lens would be a factor two or four closer. Because the volume of available lenses scales $\propto D_{L}^{3}$ (thus $\propto v_{\perp}^{3}$ ), the disk and thick disk scenarios are about equally likely, and the halo scenario is less likely than the combination of the disk scenarios by a factor of approximately four. We normalize our analysis to the thick disk scenario, keeping in mind that the 
Table 6

Parameters for 3L1S Models

\begin{tabular}{|c|c|c|c|c|}
\hline & \multicolumn{4}{|c|}{$3 \mathrm{~L} 1 \mathrm{~S}$} \\
\hline & close-close & close-wide & wide-close & wide-wide \\
\hline$\overline{\chi^{2} / \mathrm{dof}}$ & $6817.60 / 6806$ & $6817.83 / 6806$ & $6813.16 / 6806$ & $6812.87 / 6806$ \\
\hline$t_{0}\left(\mathrm{HJD}^{\prime}\right)$ & $7199.970 \pm 0.004$ & $7199.967 \pm 0.004$ & $7199.969 \pm 0.004$ & $7199.970 \pm 0.004$ \\
\hline$u_{0}$ & $0.063 \pm 0.004$ & $0.063 \pm 0.003$ & $0.062 \pm 0.004$ & $0.066 \pm 0.002$ \\
\hline$t_{\mathrm{E}}$ & $4.650 \pm 0.227$ & $4.695 \pm 0.206$ & $4.790 \pm 0.221$ & $4.485 \pm 0.111$ \\
\hline$s$ & $0.845 \pm 0.010$ & $0.840 \pm 0.010$ & $1.294 \pm 0.013$ & $1.302 \pm 0.012$ \\
\hline$q\left(10^{-3}\right)$ & $2.508 \pm 0.222$ & $2.611 \pm 0.228$ & $2.667 \pm 0.238$ & $2.959 \pm 0.225$ \\
\hline$\alpha$ & $0.906 \pm 0.009$ & $0.906 \pm 0.008$ & $0.910 \pm 0.008$ & $0.916 \pm 0.007$ \\
\hline$\rho_{*}\left(10^{-3}\right)$ & $0.552 \pm 0.123$ & $0.895 \pm 0.123$ & $0.683 \pm 0.118$ & $0.617 \pm 0.129$ \\
\hline$s_{2}$ & $1.033 \pm 0.008$ & $1.070 \pm 0.009$ & $1.020 \pm 0.009$ & $1.054 \pm 0.008$ \\
\hline$q_{2}\left(10^{-5}\right)$ & $1.950 \pm 0.862$ & $2.199 \pm 0.868$ & $1.638 \pm 0.743$ & $2.179 \pm 0.643$ \\
\hline$\psi$ & $-0.022 \pm 0.003$ & $-0.020 \pm 0.003$ & $-0.022 \pm 0.003$ & $-0.023 \pm 0.003$ \\
\hline$f_{s}(\mathrm{OGLE})$ & $0.060 \pm 0.004$ & $0.060 \pm 0.003$ & $0.059 \pm 0.004$ & $0.063 \pm 0.002$ \\
\hline$f_{b}(\mathrm{OGLE})$ & $0.096 \pm 0.004$ & $0.096 \pm 0.003$ & $0.097 \pm 0.003$ & $0.092 \pm 0.002$ \\
\hline
\end{tabular}

masses and distance could be higher or lower by a factor approximately two, depending on lens population.

A nearby lens in turn implies a low-mass lens. From Equation (1), the total lens mass (essentially the host mass) is $M=\theta_{\mathrm{E}}^{2} / \kappa \pi_{\mathrm{rel}} \simeq \theta_{\mathrm{E}}^{2} v_{\perp} / \kappa \mu_{\mathrm{rel}} \simeq 0.024 M_{\odot}\left(v_{\perp} / 100 \mathrm{~km} \mathrm{~s}^{-1}\right)$.

Thus, regardless of which population the lens system lies in (halo, thick disk, or thin disk), the host is a low-mass BD. The planet therefore has mass $m_{p}=q M \sim 20 M_{\oplus}$, i.e., slightly heavier than Neptune. Again, we should keep in mind that this value could be a factor two higher or lower if the lens lay in the halo or thin disk, respectively.

In this section, we have performed the calculations for the "close" solution given in Tables 2 and 5 because the "wide" solutions is disfavored by $\Delta \chi^{2}=15$. The "wide" solution gives qualitatively similar answers. We note, however, that the wide solution has a proper motion that is larger by a factor 1.4 and so is even more extreme.

\section{3. $3 L 1 S$}

The host-planet system in $3 \mathrm{~L} 1 \mathrm{~S}$ is very similar to the one in $2 \mathrm{~L} 2 \mathrm{~S}$. As seen from Table 2, all four solutions have qualitatively similar $\chi^{2}$ values. We trace the calculation for the wide-wide solution, which has the best $\chi^{2}$.

For $3 \mathrm{~L} 1 \mathrm{~S}$, there is of course only one source. We find $\theta_{*}=0.73 \mu$ as. Combined with $\rho=0.84 \pm 0.14 \times 10^{-3}$, this yields $\theta_{\mathrm{E}}=0.87 \pm 0.15$ mas, and $\mu=68 \pm 13 \mathrm{mas} \mathrm{yr}^{-1}$. Normalizing to the "thick disk" $\left(v_{\perp}=100 \mathrm{~km} \mathrm{~s}^{-1}\right)$ case, we find $D_{L} \sim 0.3 \mathrm{kpc}$ and $M=0.021 M_{\odot}$, and $m_{p}=q M=$ $21 M_{\oplus}$. That is, very similar to $2 \mathrm{~L} 2 \mathrm{~S}$.

However, for 3L1S, there is also a third body with mass (normalized again to the "thick disk" case), $m_{m}=0.15 M_{\oplus}$. From Figure 4, this third mass lies projected close to the second, and so could possibly be its "moon," with mass ratio $q_{3} / q_{2}=0.0076$, i.e., about a factor 1.6 smaller than the Moon/ Earth mass ratio. We discuss the issues related to such an inference in Section 6.

\section{Resolution of the Degeneracy}

The degeneracy reported here is basically a "multiplicity" of the one first pointed out by Gaudi (1998) between planetary 2L1S solutions and 1L2S solutions, particularly those with a close source approach to a faint secondary. The most secure way to distinguish between these two interpretations is to measure the color difference between the two (putative) sources. Because microlensing events involving a single source are basically achromatic, ${ }^{24}$ an evolution of apparent source color during an event is an ironclad indicator of multiple sources.

Of course if the two (or multiple) sources happen to have the same color, then their combined, magnified light will also have this color. However, in the present case, the secondary is two magnitudes fainter than the primary in the $2 \mathrm{~L} 2 \mathrm{~S}$ case, and the two sources are 4.5 and 5.5 mag fainter than the primary in the 1L3S case. The primary lies $\Delta I \sim 4.5$ mag below the clump, making it a fairly red (probably unevolved) main-sequence star. Given the observed flux ratios $q_{F, 2}$ and $q_{F, 3}$ this in turn implies, a rough color offset $\Delta(V-I)_{2}=-2.5 \log (0.189) / 2.4=0.75$ between the primary and secondary for $2 \mathrm{~L} 2 \mathrm{~S}$, and $\Delta(V-I)_{2}=$ $1.9, \Delta(V-I)_{3}=2.3$ for the secondary and tertiary in 1L3S.

Both the OGLE and KMTNet surveys routinely take $V$-band measurements. However, because the fundamental purpose of these measurements is to measure the source color (primarily in order to determine $\theta_{*}$ ), the cadence of these observations is set to obtain a few magnified points for the case of a relatively "short" event (which might be a few hours to a few days depending on field being observed). As a result, in 2015, KMTNet obtained $1 / 6$ points in $V$ band from KMTC and no in $V$ band from either KMTS or KMTA. Hence, because the anomaly was only observable by KMTA, there were no $V$ data that could probe the color of the anomalous part of the light curve.

However, incorporating MOA data can potentially yield the necessary color information. The difference between MOA's broad $R / I$ filter $\left(R_{\text {moa }}\right)$ and standard $I$ band (used by KMTNet) is much smaller than the difference between $V$ and $I$. The exact value is field dependent; however, for example, Gould et al. (2010) found that for the field of MOA-2007-BLG-192, the difference was $\Delta\left(R_{\text {moa }}-I\right)=0.26 \Delta(V-I)$. Thus, we expect that if $1 \mathrm{~L} 3 \mathrm{~S}$ is the correct model, and in the approximation that the flux normalization of the MOA data is completely set by the portions of the light curve away from the anomaly (where the total flux is dominated by the primary source, which is relatively blue) then the MOA light curve should be substantially fainter than the KMTA light curve in

\footnotetext{
${ }^{24}$ With a very modest exception when the source is resolved during a caustic crossing.
} 
the region of the anomaly. On the other hand, if $3 \mathrm{~L} 1 \mathrm{~S}$ is correct, then we expect that the two light curves should be everywhere comparable. This would be true of any $3 \mathrm{~L} 1 \mathrm{~S}$ model, so this test could rule out all $3 \mathrm{~L} 1 \mathrm{~S}$ models, even if we have not found all possible 3L1S solutions.

The case of $2 \mathrm{~L} 2 \mathrm{~S}$ should be qualitatively similar to $3 \mathrm{~L} 1 \mathrm{~S}$ because the excess light for the main anomaly is due to the primary source passing close to a caustic. Hence, this mainanomaly region should have basically the same color as the overall light curve. While we do expect the color to turn redder in the immediate neighborhood of the spike, where the secondary contributes of order half the light, unfortunately MOA does not have data during this spike.

Thus, we have a strong test that can distinguish the 1L3S solution from the $2 \mathrm{~L} 2 \mathrm{~S}$ and $3 \mathrm{~L} 1 \mathrm{~S}$ solutions: either the MOA data will show a color-dependent effect or they will not. With these predictions in mind, we incorporate MOA data into all fits, with results shown in Tables 3-6 and Figures $6-8$. We note that for the 1L3S solutions, we assume that the two fainter sources have the same flux. This is because (in contrast to the $I$ band data) the MOA data do not cover the short spike at $\mathrm{HJD}^{\prime}=7200.20$, and so do not distinguish between the two fainter sources. Moreover, for the 2L2S solutions, we impose $q_{F R}<q_{F}$, as discussed below.

Figure 6 is in agreement with the main prediction of the 1L3S model: the MOA data lie significantly below the KMTA data during the entire latter part of the night, as expected, when the two faint, red sources contribute a major part of the total flux. According to the above predictions one would also expect that the MOA data would lie below the KMTA data during the first half of the peak night, albeit by substantially less, because the two faint, red sources contribute somewhat to the total light in this portion. Instead, the MOA data are coincident or slightly above the KMTA data. However, this discrepancy can be explained by the relatively noisy character of the MOA data on non-peak nights. This leaves some freedom for the model to adjust the primary source flux to better fit the data over the anomaly. If the data were better, one would expect the primary source flux to be entirely set by the $I$-band dominated model during the epochs when the (relatively blue) primary completely dominates the light curve. Given this, the test really only predicts that the MOA data will lie further below KMTA data at the end of the night than in the beginning.

Figures 7 and 8 are also in accord with the predictions of the $1 \mathrm{~L} 3 \mathrm{~S}$ model and contradict, respectively, the $2 \mathrm{~L} 2 \mathrm{~S}$ and $3 \mathrm{~L} 1 \mathrm{~S}$ models. First, as predicted, these two figures look qualitatively similar. Second the models for MOA data track the $I$-band models, while the MOA data are systematically higher than the model at the beginning of the night and lower at the end. That is, the models have no way to accommodate the observed change in color from the beginning to the end of the night. We note that the models "try" to accommodate this change by making the secondary bluer than the primary in the 2L2S model, i.e., $q_{F R}>q_{F}$. Because the secondary is $2 \mathrm{mag}$ fainter than the primary and both are on the main sequence, we prohibit this unphysical tendency by imposing a boundary in the chains.

Comparing the best $\chi^{2}$ values for each solution (two for 1L3S, two for $2 \mathrm{~L} 2 \mathrm{~S}$, four for $3 \mathrm{~L} 1 \mathrm{~S}$ ), we see that the $1 \mathrm{~L} 3 \mathrm{~S}$ solution is preferred by $\Delta \chi^{2}=7.5$ for 2 dof over $2 \mathrm{~L} 2 \mathrm{~S}$ and by $\Delta \chi^{2}=11.9$ for 4 dof over 3L1S. These have formal probabilities of $\exp \left(-\Delta \chi^{2} / 2\right)=2.3 \%$ and $\left(\Delta \chi^{2} / 2+1\right) \exp \left(-\Delta \chi^{2} / 2\right)=$ $3.4 \%$, respectively.
Even though these $p$-values are not extremely low, we consider the degeneracy to be clearly resolved in favor of $1 \mathrm{~L} 3 \mathrm{~S}$. This work began by investigating OGLE and KMTNet data because these were all that appeared to be available. We then made special efforts to recover the MOA data, solely to test whether there was color evolution as predicted by one of the degenerate models and not by the others. Hence, because we have asked a very simple, one parameter question of the MOA data, we consider it reasonable that the above $p$-values should be taken at face value.

\section{Discussion}

The analysis of OGLE-2015-BLG-1459 lays bare both the promise and the challenges of exo-moon research using microlensing. On the one hand, it serves as a proof of concept: if there had been a three-body, BD-host/Neptune/Mars lens system present, we would have detected it. Moreover, we would have been able to demonstrate that the $3 \mathrm{~L} 1 \mathrm{~S}$ and $2 \mathrm{~L} 2 \mathrm{~S}$ solutions were preferred over the 1L3S solution. This would have left an ambiguity between $3 \mathrm{~L} 1 \mathrm{~S}$ and $2 \mathrm{~L} 2 \mathrm{~S}$, but as we briefly mention below, this could have been resolved by follow-up spectroscopy.

On the other hand, this event also illustrates two major difficulties confronting microlensing exo-moon studies, one that is practical and the other that is of a more fundamental character.

The practical problem is that microlensing experiments do not take alternate band (usually $V$-band) data often enough to measure a color change for an exo-moon. Measuring the color of a "short" event requires taking data in two different bands during that event. Exactly what is meant by "short" varies depending on the application, and may be as short as a few hours for the planets targeted by current surveys. However, in the case of this exo-moon candidate, "short" corresponds to a single data point. If the rest of the light curve had proved achromatic, a color could only have been reliably measured by alternating bands between observations. Such observations would be required to distinguish between the $2 \mathrm{~L} 2 \mathrm{~S}$ and $3 \mathrm{~L} 1 \mathrm{~S}$ solutions (had they been viable).

However, from the standpoint of a microlensing experiment focused on finding planets rather than moons (i.e., all current experiments), alternating observations between two bands would be extremely wasteful. These experiments take of order $10^{12}$ photometric measurements per year. While there may be some real microlensing events that occur on timescales comparable to the cadence (i.e., consisting of only a single point), there is no way to identify them among the enormous number of cosmic ray events and other image artifacts that occur on the same timescales. Therefore, there is no need to measure their colors, and so no need for a second band of observations on that timescale. Moreover, given the high reddening in typical microlensing fields, typical sources yield 5-10 times fewer photons in $V$ than $I$ for the same exposure time. Hence, attempting to get one $V$ for each $I$ measurement would greatly undermine the overall experiment.

In contrast, there are only a few dozen microlensing planets discovered per year and each planet is typically characterized by a few dozen data points. Hence, there are only of order $10^{3}$ points that could potentially be sensitive to exo-moons. The handful of these points that show a potential signatures can easily be vetted by examining the images (as we in fact did in this case). Hence, from the standpoint of finding exo-moons, equally dense $V$ and $I$ measurements would not be at all wasteful.

One possible compromise would be to have intensive $V$-band measurements only in a small fraction of the sky-area covered, 

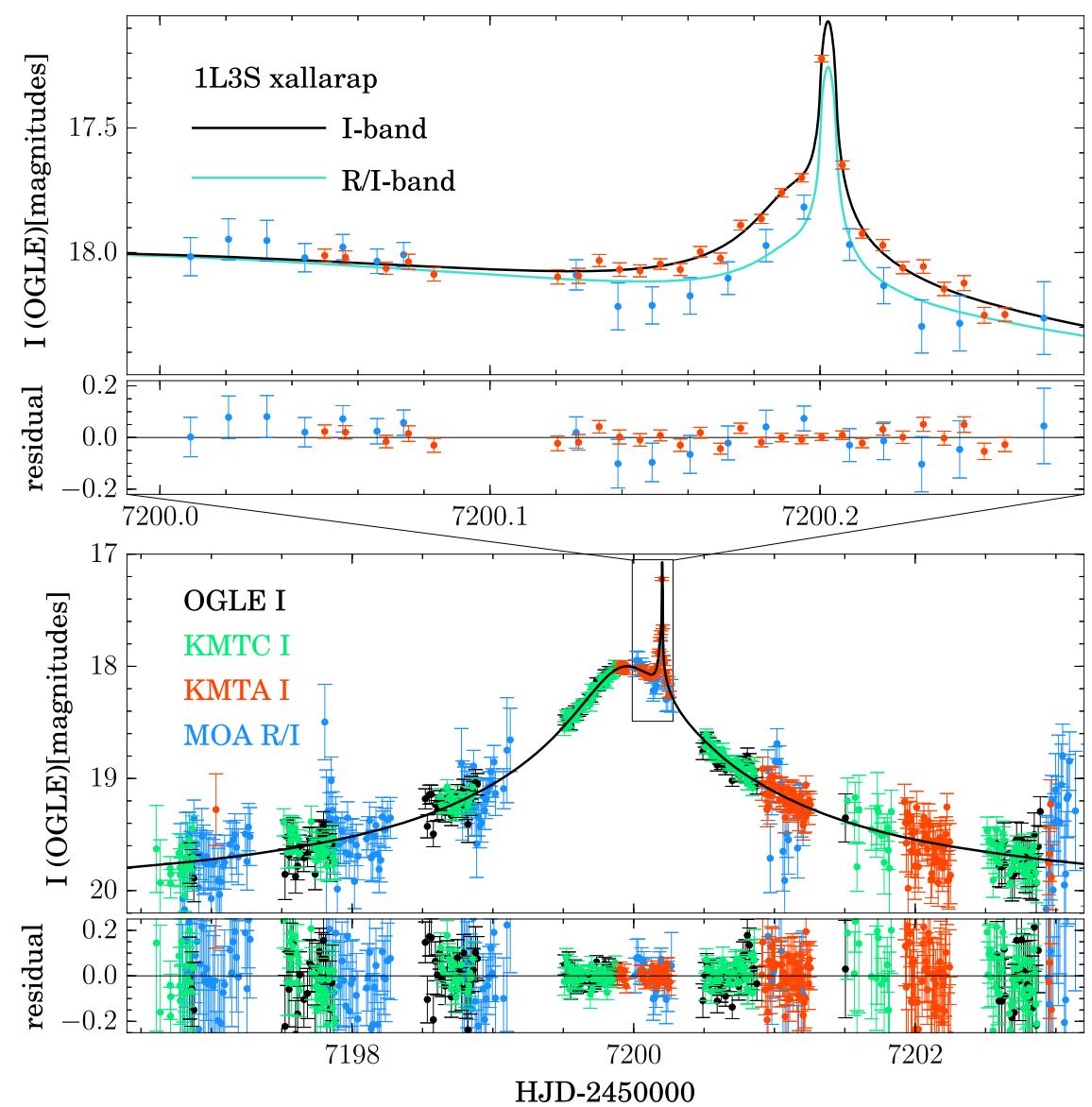

Figure 6. Joint fit of OGLE, KMTNet, and MOA data for the $1 \mathrm{~L} 3 \mathrm{~S}$ xallarap solution. Because the MOA $(R / I)$ passband is bluer than the OGLE and KMTNet $I$-band, there is an additional photometric degree of freedom, $q_{F R}$, the flux ratio of the second and third sources (assumed the same) to the primary. In the region where these sources dominate, near 7200.2, the MOA data fall below the KMTA data, as one would expect if this were the correct model. In principle, one would expect the MOA data to also fall below KMTA earlier in the night, near 7200.05, where the secondary and tertiary sources play a significant role. However, the quality of the MOA data in other magnified portions of the light curve (lower panel) is too noisy to permit strict alignment of the flux scale based on this part of the light curve alone. Hence, most of the test comes from comparison of the early part of the anomalous night relative to the later part.

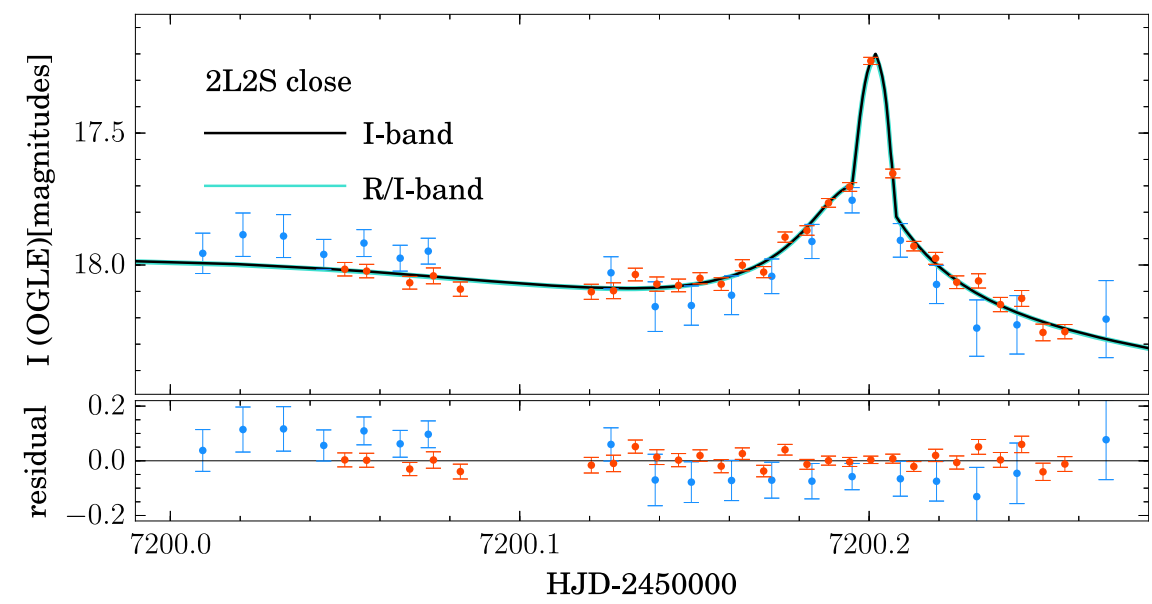

Figure 7. Joint fit of OGLE, KMTNet, and MOA data for the $2 \mathrm{~L} 2 \mathrm{~S}$ solution. The offset of the MOA and KMTA points at the beginning relative to the end of the night is, of course, the same as in Figure 6. However, the model cannot account for this as well because the secondary only dominates the light in the immediate neighborhood of the spike, where there are few MOA data points. (The zoom-out of the full light curve is not shown because it is indistinguishable from the lower panel of Figure 6.)

in particular the area with the highest number of events. For example, KMTNet currently spends one-fourth of its time on the highest density field (BLG02 + BLG42). If this field were covered $V: I$ as $1: 1$ or $1: 2$, then this would reduce the overall cadence of the experiment by factors of four-fifths or eight-ninths, respectively. This might be an acceptable cost for probing new parameter space.

The second challenge is more fundamental. If the $3 \mathrm{~L} 1 \mathrm{~S}$ solution had been the correct interpretation, then because the two smaller bodies are projected close to each other on the sky, 


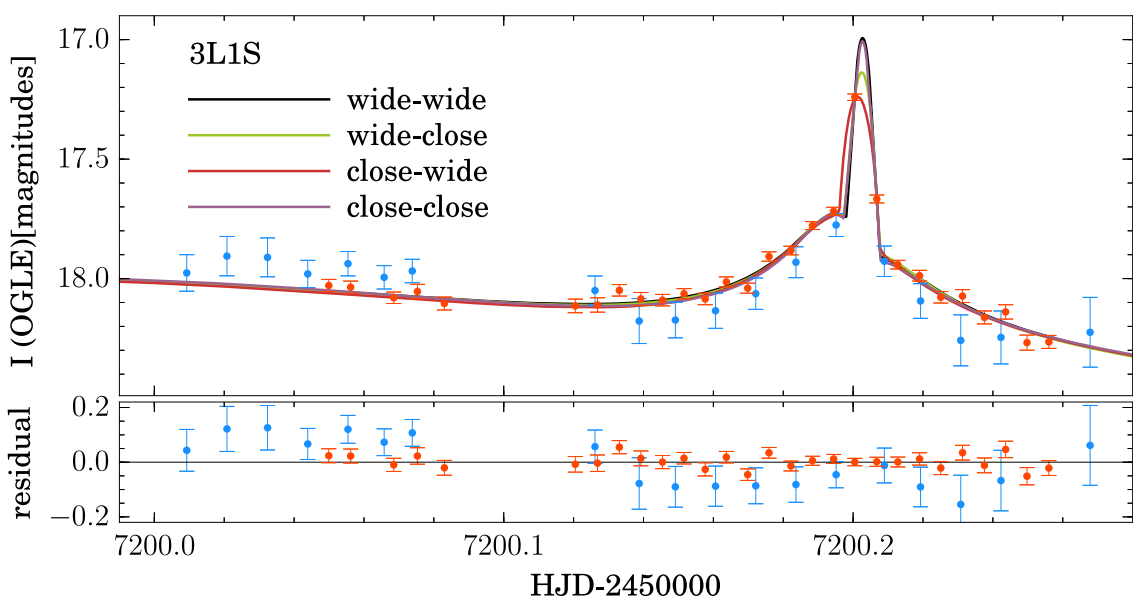

Figure 8. Joint fit of OGLE, KMTNet, and MOA data for the 3L1S solution. In this case, there is only one source, so the MOA data should should track the KMTA data. Hence, there is no way to accommodate, within the model, the fact that they are brighter in the beginning of the night relative to the end. (The zoom-out of the full light curve is not shown because it is indistinguishable from the lower panel of Figure 6.)

the Mars-class body could have been a moon of the Neptuneclass body. If it were a moon, then there are exactly three things that can be said about this planet-moon system based on the microlensing light curve. First, their host would have been a low-mass BD. Second, their mass ratio would have been about 100:1. Third, the plane of the planet-moon orbit would have been significantly misaligned from the plane of BD-planet orbit. To be bound, the moon must lie in the planet's Hill sphere, which has radius $a(q / 3)^{1 / 3}$, where $a$ is the semimajor axis. Hence, if the orbits were coplanar, then a bound orbit requires $\left(1-s_{2} / s\right)(q / 3)^{-1 / 3}<1$. In fact, assuming coplanar orbits, this ratio is 1.9 for the wide-wide case and higher for all others. However, if the planet-moon system were seen roughly face-on while the star-planet axis was inclined by at least $\theta>\tan ^{-1}(1.9)>62^{\circ}$ (as for the regular moons of Uranus) then the system would satisfy this condition.

However, it also would have been possible that the third body was not bound to the Neptune-class planet, and in fact independently orbited the star on a wider or closer orbit. Because of the nature of the microlensing technique, we can detect only the projected positions of these two bodies and cannot generally tell whether they are in front of or behind the plane of the lens. Therefore, it may be that we would happen by chance to observe the system at a point in the orbits of two planets that makes them appear to be close to each other in projection even though they orbit the host independently. Unfortunately, there is no way to distinguish between these possibilities based on the microlensing data. Nor would there be any possibility of further investigating the system with present, or presently conceived, instruments.

Moreover, there will always be this ambiguity even in cases for which the third body lies projected within the Hill sphere of the second. It may seem more likely that two bodies projected close together would be bound to each other in a planet-moon system: two bound bodies will always appear close in projection because they are physically close. The alternative requires that we have observed the two planets at a special time in their orbits by chance. However, we must also take into account observational bias. Even if the two planetary bodies are not physically related, the probability of detectable signals from both bodies is increased if they appear close together in projection. Both bodies will preferentially be found close to the star's Einstein radius, and the probability of detecting a small third body will be enhanced by its proximity (in projection) to the second body. Thus, the study of microlensing exo-moons must be done on a statistical basis and will also require systematic simulations to quantify this observational bias. At the next level of complexity, such simulations should take account of dynamical interactions, such as Lidov-Kozai oscillations, which might affect the stability of marginally Hill-stable systems.

These issues must be taken into account not only in existing ground-based surveys like OGLE and KMTNet but also in the future WFIRST microlensing survey (Spergel et al. 2013). WFIRST's precise photometric precision makes it potentially far more capable of detecting the subtle signals from exomoons as compared to present surveys. This work suggests that such studies would benefit from frequent data in a second band to distinguish additional lens planets or moons from additional source stars. In addition, to more fully characterize very short perturbations with $t_{*} \equiv \rho t_{\mathrm{E}} \lesssim 9$ minutes requires a faster cadence. This might be accomplished by a tiered observing strategy, such as the one adopted by OGLE long ago in which some fields are observed at a very high cadence at the expense of other fields. In fact, in 2016 KMTNet changed its strategy to follow the same principle.

Finally, for completeness, we note that if the MOA data had strongly preferred the (2L2S or $3 \mathrm{~L} 1 \mathrm{~S})$ solutions instead of $1 \mathrm{~L} 3 \mathrm{~S}$, then the remaining ambiguity could have been resolved by follow-up spectroscopy. For $2 \mathrm{~L} 2 \mathrm{~S}$, the secondary is only about $1.6 \mathrm{mag}$ fainter than the primary and is likely moving at several tens of kilometers per second relative to it. Hence, it could be separately detected with an $R=20,000$ spectrograph. While the second source would be quite faint by today's standards, $I \sim 23$, such spectroscopy would be in the range of next generation (" $30 \mathrm{~m}$ ") class telescopes.

From OGLE-2015-BLG-1459, we can conclude that exomoon studies with microlensing will be challenging. Although we ultimately rejected the exo-moon explanation for this event, the event provided the first practical glimpse of what is required to meet these challenges. 
Work by K.H.H. was support by KASI grant 2017-1-830-03. Work by W.Z., Y.K.J., and A.G. were supported by AST1516842 from the US NSF. W.Z., I.G.S., and A.G. were supported by JPL grant 1500811 . This research has made use of the KMTNet system operated by the Korea Astronomy and Space Science Institute (KASI) and the data were obtained at three host sites of CTIO in Chile, SAAO in South Africa, and SSO in Australia. Work by C.H. was supported by the grant (2017R1A4A101517) of National Research Foundation of Korea. The OGLE Team thanks Prof. G. Pietrzyński for his contribution to the collection of the OGLE photometric data. The OGLE project has received funding from the National Science Centre, Poland, grant MAESTRO 2014/14/A/ST9/ 00121 to AU. The MOA project is supported by JSPS KAKENHI Grant Number JSPS24253004, JSPS26247023, JSPS23340064, JSPS15H00781, and JP16H06287.

\section{ORCID iDs}

K.-H. Hwang (D) https://orcid.org/0000-0002-9241-4117

M. D. Albrow (i) https://orcid.org/0000-0003-3316-4012

C. Han (ib https://orcid.org/0000-0002-2641-9964

Y.-H. Ryu (iD https://orcid.org/0000-0001-9823-2907

I.-G. Shin (i) https://orcid.org/0000-0002-4355-9838

J. C. Yee (ib https://orcid.org/0000-0001-9481-7123

R. W. Pogge (ib https://orcid.org/0000-0003-1435-3053

R. Poleski (i) https://orcid.org/0000-0002-9245-6368

J. Skowron (ii) https://orcid.org/0000-0002-2335-1730

P. Pietrukowicz (iD https://orcid.org/0000-0002-2339-5899

D. P. Bennett (iD https://orcid.org/0000-0001-8043-8413

A. Fukui (i) https://orcid.org/0000-0002-4909-5763

Y. Itow (iD https://orcid.org/0000-0002-8198-1968

K. Kawasaki (iD https://orcid.org/0000-0003-2006-1735

N. Koshimoto (iD https://orcid.org/0000-0003-2302-9562
S. Miyazaki (ib https://orcid.org/0000-0002-1962-904X

N. J. Rattenbury (i) https://orcid.org/0000-0001-5069-319X

D. Suzuki (iD https://orcid.org/0000-0002-5843-9433

\section{References}

Alard, C., \& Lupton, R. H. 1998, ApJ, 503, 325

Albrow, M. D., Horne, K., Bramich, D. M., et al. 2009, MNRAS, 397, 2099

Bensby, T., Yee, J. C., Feltzing, S., et al. 2013, A\&A, 549A, 147

Bessell, M. S., \& Brett, J. M. 1988, PASP, 100, 1134

Bond, I. A., Bennett, D. P., Sumi, T., et al. 2017, MNRAS, 469, 2434

Chung, S.-J., \& Ryu, Y.-H. 2016, ApJ, 626, 90

Daněk, K., \& Heyrovský, D. 2015a, ApJ, 806, 63

Daněk, K., \& Heyrovský, D. 2015b, ApJ, 806, 99

Dominik, M. 1999, A\&A, 349, 108

Fabrycky, D., \& Tremaine, S. 2007, ApJ, 669, 1298

Gaudi, B. S. 1998, ApJ, 506, 533

Gaudi, B. S. 2012, ARA\&A, 50, 411

Gould, A., Dong, S., Bennett, D. B., et al. 2010, ApJ, 710, 1800

Griest, K., \& Safizadeh, N. 1998, ApJ, 500, 37

Han, C. 2008, ApJ, 684, 684

Han, C., \& Han, W. 2002, ApJ, 560, 490

Han, C., Udalski, A., Choi, J.-Y., et al. 2013, ApJL, 762, L28

Han, C., Udalski, A., Gould, A., et al. 2017, AJ, 154, 133

Han, C., Udalski, A., Gould, A., et al. 2017, AJ, 154, 223

Kervella, P., Thévenin, F., Di Folco, E., \& Ségransan, D. 2004, A\&A, 426, 297

Kim, S.-L., Lee, C.-U., Park, B.-G., et al. 2016, JKAS, 49, 37

Kipping, D. M., Schmitt, A. R., Huang, X., et al. 2015, ApJ, 813, 14

Liebig, C., \& Wambsganss, J. 2010, A\&A, 520A, 68

Mróz, P., Han, C., Udalski, A., et al. 2017, AJ, 153, 143

Nataf, D. M., Gould, A., Fouqué, P., et al. 2013, ApJ, 769, 88

Song, Y.-Y., Mao, S., \& An, J. H. 2014, MNRAS, 437, 4006

Spergel, D. N., Gehrels, N., Breckinridge, J., et al. 2013, arXiv:1305.5422

Teachey, A., Kipping, D. M., \& Schmitt, A. R. 2018, AJ, 155, 36

Udalski, A., Szymanski, M., Kaluzny, J., et al. 1994, AcA, 44, 317

Udalski, A., Szymański, M. K., \& Szymański, G. 2015, AcA, 65, 1

Woźniak, P. R. 2000, AcA, 50, 421

Yee, J. C., Shvartzvald, Y., Gal-Yam, A., et al. 2012, ApJ, 755, 102

Yoo, J., DePoy, D. L., Gal-Yam, A., et al. 2004, ApJ, 603, 139 